\title{
Research on Ontology-based Catering Recommendation Knowledge Model
}

\section{Tang Dongping*, Zhu Xiangyu, Zhu Weiquan}

College of Business Administration, South China University of Technology, Guangzhou, China

\section{Email address:}

653473738@qq.com (Tang Dongping),1154135932@qq.com (Zhu Xiangyu),865807189@qq.com (Zhu Weiquan)

*Corresponding author

\section{To cite this article:}

Tang Dongping, Zhu Xiangyu, Zhu Weiquan. Research on Ontology-based Catering Recommendation Knowledge Model. Science Discovery. Vol. 7, No. 5, 2019, pp. 373-378. doi: 10.11648/j.sd.20190705.29

Received: October 28, 2019; Accepted: November 23, 2019; Published: December 3, 2019

\begin{abstract}
At present, the healthy eating information available on the Internet is growing rapidly, but it is very time-consuming for users to understand healthy eating through Internet data. This article starts from the relevant theories of TCM constitution and diet, and uses the methods and tools of ontology modeling to carry out food recommendation knowledge. Modeling, constructing a knowledge model including the user ontology and the ontology of the healthy catering field, using Protégé software to realize the establishment of the ontology, forming a standardized representation of the catering ontology, establishing a user ontology model and a "healthy catering domain ontology model", which makes the knowledge related to catering recommendation easy to reconstruct and expand.In the meantime,the model makes it easy to expand the knowledge and build the knowledge map , providing a theoretical basis for healthy diet recommendation services.
\end{abstract}

Keywords: Diet, Ontology, Recommendation

\section{基于本体的餐饮推荐知识模型研究}

唐东平", 朱向宇, 朱伟权

华南理工大学工商管理学院, 广州, 中国

\section{邮箱}

653473738@qq.com (唐东平), 1154135932@qq.com（朱向宇）,865807189@qq.com(朱伟权)

摘要：目前互联网上可获得的健康饮食信息正在迅速增长，但用户通过互联网数据了解健康饮食非常耗时，本文从中 医体质学以及食疗的相关理论出发, 利用本体建模的方法与工具, 进行餐饮推荐知识建模, 构造出包括用户本体和健 康餐饮领域本体的知识模型, 使用Protégé软件实现本体的建立, 形成餐饮本体的规范化表示, 建立出用户本体模型以 及“健康餐饮领域本体模型”, 使得餐饮推荐相关的知识便于重构以及拓展, 并且便于知识的扩展和知识图谱的构建, 为健康饮食推荐服务提供理论指导。

关键词: 餐饮, 本体, 推荐 


\section{1. 引言}

近代以来, 对体质进行系统专门的研究起始于 20 世 纪70年代, “体质”是在中医理论发展过程中形成的病理 生理学概念。经过大量的中医专家学者研究, 并且对临 床实践的归纳, 在 2009 年中华中医协会发布了一份中医 体质判断的标准文件《中医体质判定与分类标准》，里 面对于中医体质学的体质类型划分做了总结, 并给出了 每种体质的判断准则, 将体质类型划分为九种不同的类 型 $[1]$ 。

这九种体质类型分别为平和质、湿热质、阴虚质、气 虚质、气郁质、阳虚质、痰湿质、血瘀质和特禀质。其中 的平和质属于理想健康的体质, 另外的八种体质类型属于 亚健康状态。

中医体质学理论认为体质是可以调节的, 具有相对稳 定性而且动态可变性的特点, 饮食营养因素是对于体质的 形成以及变化有着重要的影响作用[2]。食疗理论通过体质 的动态可变性来对亚健康的体质状态进行调整, 根据不同 体质的饮食需求来弥补当前体质状态中某些特征的不足, 促使体质向着健康的平和体质状态转变, 进而改善亚健康 体质状态[3]。中医食疗理论的形成离不开中医研究者在实
践过程中对于食物作用的深入理解, 总结出食物中包含寒、 凉、平、温、热这五种不同的性质, 以及五味等等的食物 属性, 食物的属性对于身体的体质状态有着影响作用[4]。 此外, 中医养生学中有着“食养食疗”的观点, 表示一些疾 病可以通过饮食来达到一定的治疗和预防效果, 并有“医 食同源”、“寓医于食”的观点。同时传统中医医学根据四 季气候的变化提出不同的饮食原则 [5]。

本体研究虽然已经开展了许多年, 但是到目前为止学 术界还没有提出构建本体的统一定论, 但本体的构建形散 而神不散。人工构建的方式通常需要由大量的领域专家相 互协作完成, 比较有代表的人工构建本体如WordNet (Miller, 1995)。目前, 对于本体的构建, 国外较为成 型的方法主要有: 骨架法、斯坦福大学的七步法、加拿大 多伦多大学的TOVE企业建模法、METHONTOLOGY框架 法等[6]。

本文的本体构建采用斯坦福大学开发的七步法, 包含 7个步骤：需求分析、可重用性考察、核心概念及重要术 语的描述、概念类的分层关系定义、概念类的属性定义、 概念类中各个属性的限制定义、实例的创建, 具体的构建 流程如图1所示。

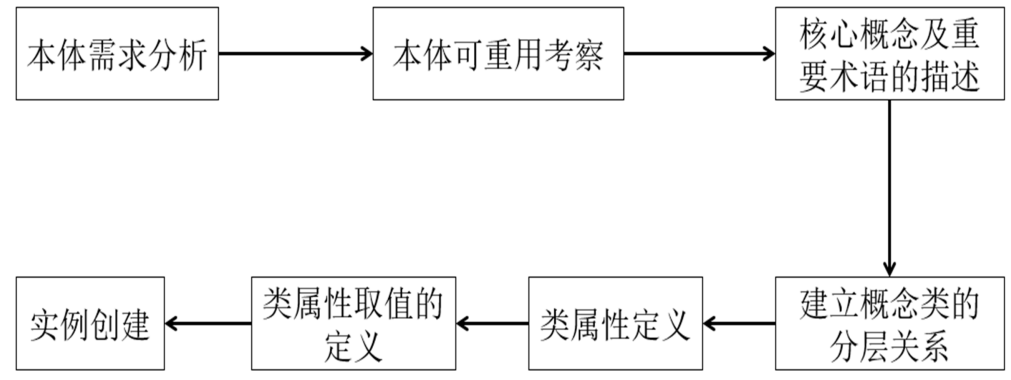

图1 基于七步法的本体构建流程。

本体构建除了有相应的构建方法之外, 还需要有构建 工具来辅助本体的构建, 本体构建工具对于本体的构建有 着非常重要的作用。随着本体研究的发展以及本体的应用 逐渐增多, 许多研究人员设计并且开发出各种各样的本体 构建工具, 用以实现本体的构建和开发工作，极大地方便 本体研究者进行本体的构建、维护, 并且本体工具的使用 也提高了构建本体的效率。本体构建工具在国外也有相当 长的研究历史, 在诸多本体构建工具中, 较为流行的工具 有马德里大学开发的WebODE、斯坦福大学研发的Protégé、 卡尔斯鲁厄大学研发的OntoEdit、曼切斯特大学研发的 OilEd等, 其中Protégé由于其易用性、稳定性以及开放源 代码的特性成为获得了众多用户的喜爱。目前Protégé己有 超过 30 万注册用户，成为了最受欢迎的本体开发工具之一 [7-8]。

通过对以往的文献调研, 在学术研究领域内针对餐饮 推荐相关的本体建模, Protégé系列工具有着较高比例的使 用, 因此本文在选择本体构建工具时选择使用Protégé软件, 采用的是最新的Protégé5.5.0版本, 便于将本文构建的本体 进行知识的共享, 提高本体模型的互用性。

\section{2. 餐饮推荐知识建模}

本文在实现饮食推荐服务的过程中需要涉及用户、 食物、健康等多方面的数据和知识, 这些不同种类的知 识和数据可以被归纳为领域的核心概念, 领域的核心概 念对应的是本体中的类（owl:Class），每个核心概念都 对应着许多实例，例如“食谱”这个概念下，就对应着“银 耳雪梨金桔美”、“鱼香茄子保”、“苦瓜炒蛋”等实例。我 们可以对餐饮推荐中的用户和健康餐饮领域建立模型, 如图2所示。

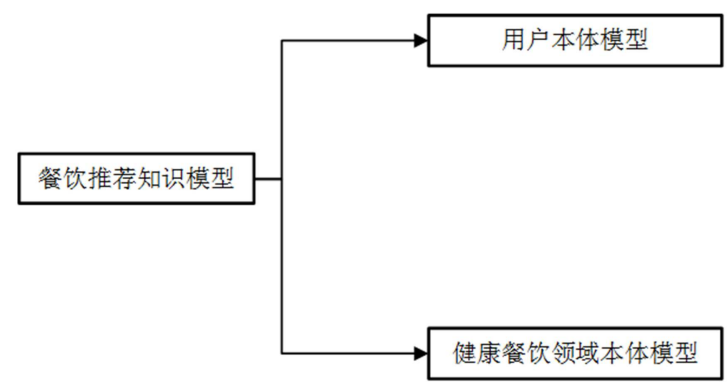

图2 餐饮推荐知识模型。 


\section{1. 用户本体模型}

餐饮推荐知识模型中的用户本体模型主要用于记录 用户的相关基本信息以及用户行为记录。引入本体论进行 用户模型的构建可以使得推荐系统发现用户的饮食需求, 从而推荐符合用户需要的餐饮。

根据餐饮推荐的需求[9], 描述与餐饮相关的用户信息, 定义的用户本体模型如下，如图3所示，包括基本信息、 健康状态、行为记录。

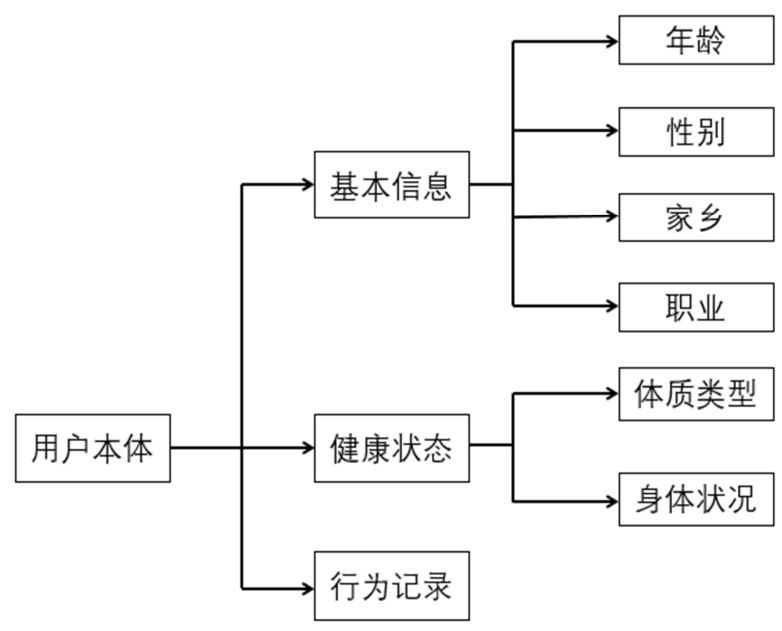

图3 用户本体模型。
其中, 这三类具体包含的内容如下:

（1）基本信息, 指用户的个人特征信息, 本文结合 餐饮推荐的需要, 选择相关的属性作为基本信息, 包括性 别、年龄、职业、地区。

（2）健康状态, 指用户的健康相关信息, 包括体质 类型、身体状况（健康或者疾病）。

（3）行为记录, 指用户进行的餐饮相关历史行为。

\section{2. 健康餐饮领域本体模型}

健康餐饮是推荐算法中的推荐对象, 对餐饮进行本体 建模是对推荐对象建模。因此, 通过参考并复用现有本体 模型, 并结合餐饮领域知识来构建领域本体, 以详细地对 餐饮相关的实体、属性和关系进行描述。我们通过相关文 献研究和专家学者以及餐饮从业专业人员调研访谈, 篮选 出一些比较重要的因素, 定义了与餐饮和食疗的主题密切 相关的核心本体概念, 包括: 食材、食谱、症状、疾病、 人群、季节、体质类型。其中, 我们将饮食需求中个体差 异抽象为人群, 比如年龄、特殊人群。

我们可以建立健康餐饮领域本体的概念模型如图4所 示, 除了给出相关的概念之外, 还定义了概念之间的关系, 图4中描述了这几个类之间的关系，例如不同的食材适合 不同的季节、体质类型、人群等，不同的食材构成不同的 食谱, 食谱是否适合某些疾病的症状以及不同的人群等。

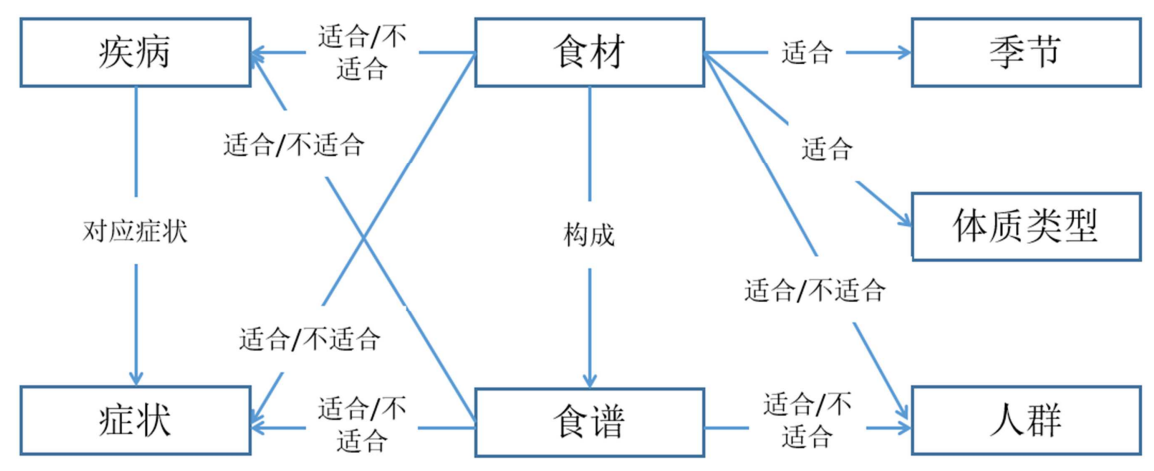

图4 健康餐饮领域本体概念模型。

在分析了健康餐饮领域的本体概念模型之后, 进一步 确定核心概念数据描述的各个实体类以及各个实体类所 具有的属性以及各个属性之间的关系。为了更好地描述食 材、食谱、症状、疾病、人群、体质类型、季节的信息， 根据上述对本体概念的分析之后可以进行对健康餐饮领 域本体中的基本类和属性进行概念的提取, 基本概念的描 述分别为:

（1）食谱类，指利用各种烹饪原料、通过各种烹调 技法制作出的食物。如“鱼香茄子煲”、“苦瓜炒蛋”等。

（2）食材类, 食材是构成食谱的原材料, 如牛肉、 排骨、红豆等等。

(3) 症状类, 指机体内出现的一些病症状态。一些 常见的症状如头晕、咳嗽、胃痛等。

(4) 疾病类, 指身体在病因的影响下, 出现的身体 异常状态, 如肾结石、糖尿病等。
（5）人群类, 人群代表一组易患相同疾病和症状的 特殊人群, 根据年龄和身体素质进行分类, 如孕妇, 老年 人, 幼儿等 $[10]$ 。

（6）体质类型类，依据中华中医药协会的体质划分 准则分为九种不同类型的体质状态，即平和质、阳虚质、 湿热质、阴虚质、气郁质、痰湿质、气虚质、血瘀质和特 禀质[3]。 冬季。

（7）季节类, 按四季划分, 即春季、夏季、秋季、

\section{3. 本体建模}

现阶段还没有形成一套完善的餐饮推荐服务体系, 其 中包括对于各种饮食数据、食谱数据、以及用户个人健康 数据的规范化描述。本研究的主要目标之一是捕获餐饮领 
域的本体知识, 以指导餐饮 $\mathrm{O} 2 \mathrm{O}$ 智能推荐模型的设计。出 于这个原因, 我们选择设计OWL本体语言来描述健康餐 饮领域的本体。

在Protégé本体建模中, 定义了 2 种类属性, 一种是数 据属性（Data properties）, 用于对类的数据值和数据类型 进行定义, 描述概念类的固有属性, 如食谱名称、食谱口 味等等。

另一种是对象属性 (Object properties), 用于对本体 模型中类与类之间的关系进行定义, 可以看成是一条连接 着两个类的边, 这条边是有指向性的, 由一个类指向另一 个类, 例如“isPartOf”是“foodMaterial”和“recipe”两个类之 间的对象属性，表示为“foodMaterial-isPartOf-recipe”, 代表食材是食谱的组成部分。

对于每种类属性的约束有定义域和值域。定义域 （domain）：用来描述哪些范围内的类可以使用该属性。 值域（range）：用来描述该属性的值可以是哪些范围。

根据前面确定的各个实体类以及各个实体类所具有 的属性以及各个属性之间的关系, 本文利用Protégé软件建 立了餐饮推荐本体模型，如图5所示。其中包含了用户本 体模型以及健康餐饮领域本体模型两部分, 在下面将会对 模型中的各个类进行解释说明。

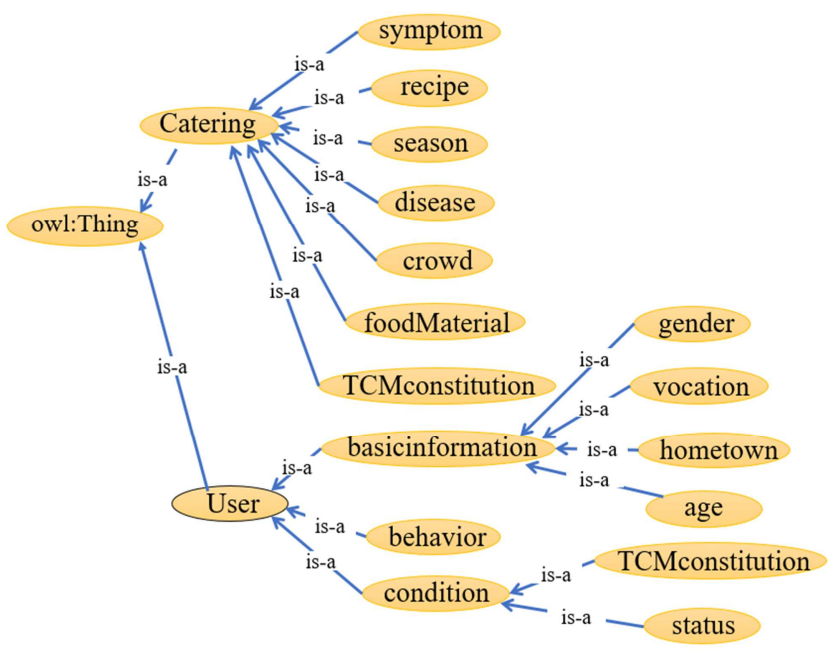

图5 餐饮推荐本体总体结构。

3.1. 用户本体

\subsection{1. 类 (class) 定义}

根据2.1中定义的用户本体模型, 建立用户本体类, 包括基本信息、行为记录、健康状态三个主要的类, 如表 1所示。

表1 用户本体类的定义。

\begin{tabular}{llll}
\hline 类标识 & 父类 & 子类 & 定义 \\
\hline basicInformation & - & Age, gender, hometown, vocation \\
behavior & - & - & 基本信息 \\
condition & - & Status, TCMconsitution & 行为记录 \\
age & basicInformation & - & 健康状态 \\
gender & basicInformation & - & 年龄 \\
hometown & basicInformation & - & 性别 \\
vocation & basicInformation & 家乡 \\
TCMconsitution & condition & - & 职业类型 \\
status & condition & 体质类型 & 身体状况 \\
\hline
\end{tabular}

如图6所示，在Protégé中建立用户本体的类，User类 中包括用户基本信息如年龄、性别、家乡、职业也包括用 户的行为记录以及一些状况如身体状况和体质类型。

\section{$\dot{\nabla}$... User

\begin{tabular}{|l|l}
\hline & basicInformation \\
age \\
gender \\
hometown \\
vocation \\
behavior \\
condition \\
status \\
TCMconstitution
\end{tabular}

图6 基于Protégé本体编辑器的用户本体模型。

\subsection{2. 类属性（Properties）定义}

在定义了类之后, 对类的属性进行定义, 如表 2 和表 3 所示。其中表 2 中列举的是对象属性, 表3中列举的是数据 属性。其中, 对象属性在某种程度上来说也是一种对概念 关系的描述。例如表2中定义域基础信息（basicinformation） 具有包含年龄（hasage）属性，取值是年龄（age），基础 信息 (basicinformation) 和年龄 (age) 是类; 表3中年龄 （age）具有年龄（Hage）属性, 取值是字符串（String）, 年龄（age）是类。

表2 用户本体类对象属性的定义。

\begin{tabular}{|c|c|c|c|}
\hline 属性标识符 & 定义域 & 值域 & 描述 \\
\hline Has Age & basicInformation & age & 包含年龄属性 \\
\hline Has Gender & basicInformation & gender & 包含性别属性 \\
\hline Has Hometown & basicInformation & hometown & 包含家乡属性 \\
\hline Has Vocation & basicInformation & vocation & 包含职业属性 \\
\hline $\begin{array}{l}\text { Has TCM } \\
\text { consitution }\end{array}$ & condition & TCM consitution & 包含体质信息 \\
\hline Has Status & condition & status & $\begin{array}{l}\text { 包含身体状况信 } \\
\text { 息 }\end{array}$ \\
\hline
\end{tabular}


表 3 用户本体类数据属性的定义

\begin{tabular}{llll}
\hline 属性标识符 & 定义域 & 值域 & 描述 \\
\hline Hage & age & String & 年龄 \\
Hgender & gender & String & 性别 \\
Hhometown & hometown & String & 家乡 \\
Hvocation & vocation & String & 职业类型 \\
HTCMconsitution & TCMconsitution & String & 体质类型 \\
Hstatus & status & String & 身体状况 \\
\hline
\end{tabular}

\section{2. 健康餐饮领域本体}

\subsection{1. 类 (class) 定义}

根据上一节中归纳的领域概念结构, 首先建立出本体 中主要的类, 包含: 食材、食谱、症状、疾病、人群、体 质类型、季节。健康餐饮领域本体类中具体类定义如表 4 所示。其中foodMaterial是食材, 没有父类和子类。同样的, 其他的类也没有父类和子类。

表4 健康餐饮领域本体类的定义。

\begin{tabular}{llll}
\hline 类标识 & 父类 & 子类 & 定义 \\
\hline foodMaterial & - & - & 食材 \\
recipe & - & - & 食谱 \\
crowd & - & - & 人群 \\
symptom & - & - & 症状 \\
disease & - & - & 疾病 \\
TCMconstitution & - & - & 体质类型 \\
season & - & - & 季节 \\
\hline
\end{tabular}

在Protégé 中建立本体的 7 个类, 结构如图7所示, Catering类中包括人群、疾病、食材、食谱、季节、症状 以及体质类型。

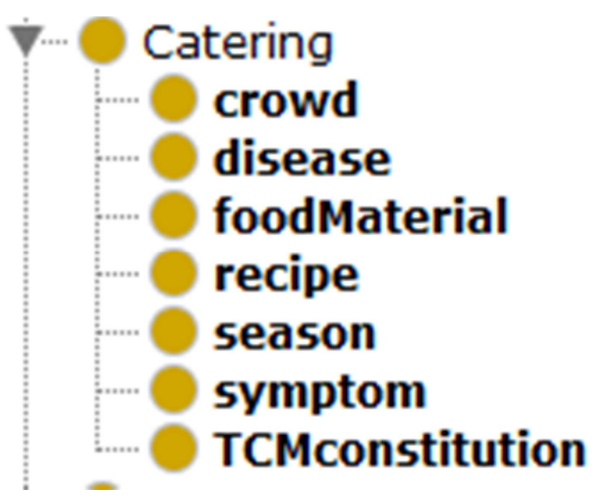

图7 基于Protégé本体编辑器的健康餐饮领域本体。

\subsection{2. 类属性 (Properties) 定义}

在定义了类之后，对类的属性进行定义，如表 5 和表 6 所示。其中, 表 5 中是关于健康餐饮领域本体的概念关系 及对象属性的定义; 表6展示了健康餐饮领域本体的概念 关系及数据属性的定义。例如表 5 中食谱（recipe）具有食 材（hasMaterial）属性, 取值是食材（foodMaterial）, 描 述的关系是食谱包含食材; 表6中食材（foodMaterial）具 有食材名称 (materialName) 属性, 取值是字符串 (String) 描述的是食材的名称。
表5 健康餐饮领域本体的概念关系及对象属性的定义。

\begin{tabular}{|c|c|c|c|}
\hline 属性标识符 & 定义域 & 值域 & 描述 \\
\hline Has Material & recipe & foodMaterial & 食谱包含食材 \\
\hline Is GoodForCrowd & recipe & crowd & $\begin{array}{l}\text { 良㬐迠合的人 } \\
\text { 群 }\end{array}$ \\
\hline Is Bad For Crowd & recipe & crowd & $\begin{array}{l}\text { 食谱不适合的 } \\
\text { 人群 }\end{array}$ \\
\hline Is Good For DS & $\begin{array}{l}\text { foodMaterial } \\
\text { recipe }\end{array}$ & $\begin{array}{l}\text { symptom } \\
\text { disease }\end{array}$ & $\begin{array}{l}\text { 对某个症状/ } \\
\text { 疾病有益的食 } \\
\text { 谱/食材 }\end{array}$ \\
\hline Is Bad For DS & $\begin{array}{l}\text { foodMaterial } \\
\text { recipe }\end{array}$ & $\begin{array}{l}\text { symptom } \\
\text { disease }\end{array}$ & $\begin{array}{l}\text { 对某个症状/ } \\
\text { 疾病不适合的 } \\
\text { 食谱/食材 }\end{array}$ \\
\hline Is Related To Symptom & disease & symptom & 疾病对应症状 \\
\hline Is Related To Season & $\begin{array}{l}\text { foodMaterial } \\
\text { recipe }\end{array}$ & season & $\begin{array}{l}\text { 食材/食谱适 } \\
\text { 合的季节 }\end{array}$ \\
\hline $\begin{array}{l}\text { Is Good For } \\
\text { Constitution }\end{array}$ & $\begin{array}{l}\text { foodMaterial } \\
\text { recipe }\end{array}$ & $\begin{array}{l}\text { TCMconstituti } \\
\text { on }\end{array}$ & $\begin{array}{l}\text { 食谱/食材适 } \\
\text { 合的体质类型 }\end{array}$ \\
\hline
\end{tabular}

表6 健康餐饮领域本体的概念关系及数据属性的定义。

\begin{tabular}{llll}
\hline 属性标识符 & 定义域 & 值域 & 描述 \\
\hline materialName & foodMaterial & String & 食材名称 \\
wuxing & foodMaterial & String & 五性 \\
wuwei & foodMaterial & String & 五味 \\
efficacy & foodMaterial & String & 功效 \\
symptomName & symptom & String & 症状名称 \\
diseaseName & disease & String & 疾病名称 \\
constitutionName & TCMconstitution & String & 体质名称 \\
seasonName & season & String & 季节名称 \\
recipeName & recipe & String & 食谱名称 \\
recipeTaste & recipe & String & 口味 \\
recipeType & recipe & String & 食谱类型 \\
recipeCookWay & recipe & String & 烹饪方法 \\
recipeEfficacy & recipe & String & 功效 \\
\hline
\end{tabular}

根据上表中对对象的对象属性的定义, 在Protégé中建 立了对象之间的 8 个关联关系, 结构如图8所示。

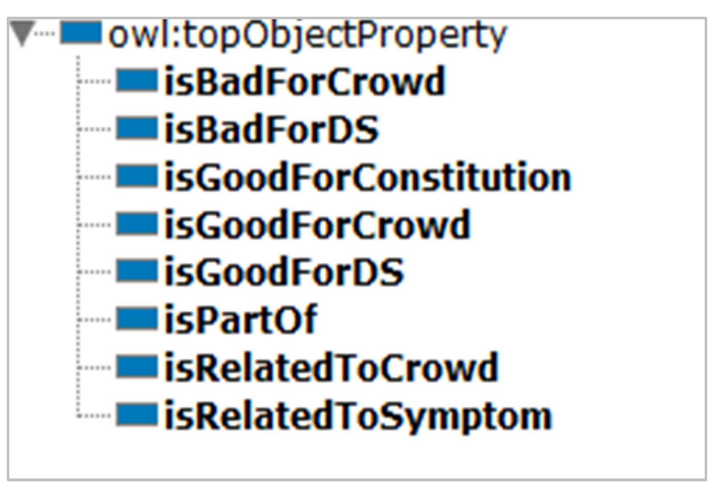

图8 Protégé中的类对象属性。

在Protégé中建立完成本体中的类和类关系之后, 各个 类之间的关联关系已经确立。类之间的关联结构如图9所 示, 通过Protégé对健康餐饮领域的各个概念类进行定义, 并将类之间的关系进行定义，提供了一个清晰的类关系可 视化图, 可为后续的本体知识架构提供基础。 


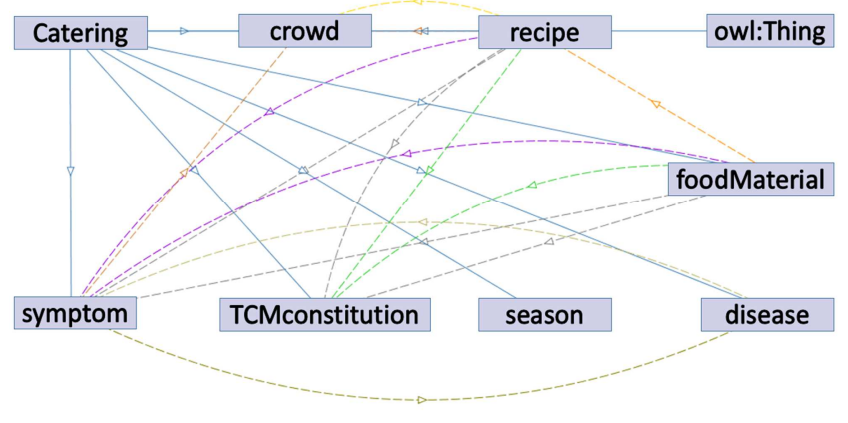

图9 健康餐饮领域本体类关系可视化图。

\section{4. 结论}

本文构建的餐饮领域本体模型实现对餐饮领域的概 念化描述, 并且引入了中国饮食知识以及中医知识, 形成 “健康餐饮领域本体模型”，进一步完善餐饮领域的知识模 型, 便于集成和整合不同类型的知识。未来我们可以改进 餐饮推荐本体模型, 增加更多的数据来源, 包括用户社交 信息、用户运动记录、可穿戴设备获取的健康指标等等, 挖掘更多与用户关联的数据跟餐饮推荐之间的关系, 提升 推荐的效果。对世界上不同地区的饮食文化进行研究, 将 更加多元的饮食文化融合到餐饮推荐中, 建立更加全面的 知识图谱, 满足各种人群的餐饮推荐需求。

\section{致谢}

本文得到广东省本科高校创新创业教育改革研究项 目（2018A090921）；华南理工大学 SRP 项目 (X201910561159) 支持。

\section{参考文献}

[1] 李东遥, 李力恒, 曹风鸣. 中医体质、中医体质分类与判定系 统 $[J]$.科学技术创新,2019(03):29-30.

[2] 陈禹, 李玲孺, 石励, et al. 基于中医体质的营养学研究方 法探讨[J]. 中华中医药学刊, 2016(3):554-556.

[3] 李婧. 基于体质调理的食疗咨询系统设计研究[D]. 中南大 学, 2014.

[4] 卢剑锋. 健康餐饮专家系统研究与实现 [D]. 华南理工大 学, 2018 .

[5] 李欢旗. 论中医饮食疗法的研究应用 [J]. 亚太传统医 药,2016,12(17):61-62.

[6] 郭叶鹏. 基于感知价值的电子商务商品本体构建方法及应 用研究[D].河北大学,2019.

[7] 郝伟学. 中医健康知识图谱的构建研究 [D]. 北京交通大 学, 2017 .

[8] 章勇, 吕俊白. 基于Protege的本体建模研究综述 [J].福建电 脑,2011,27(01):43-45.

[9] 吕苗. 基于情境的商品个性化推荐方法研究[D]. 大连理工 大学, 2015 .

[10] Chi Y, Yu C, Qi X, et al. Knowledge Management in Healthcare Sustainability: A Smart Healthy Diet Assistant in Traditional Chinese Medicine Culture [J]. Sustainability, 2018, 10 (11): 4197. 